\title{
EVALUATION OF A LOW-COST, PC-BASED DRIVING SIMULATOR TO ASSESS PERSONS WITH COGNITIVE IMPAIRMENTS DUE TO BRAIN INJURY
}

\author{
Jerry Wachtel \\ The Veridian Group, Inc. \\ William K. Durfee \\ Mechanical Engineering \\ University of Minnesota \\ Theodore J. Rosenthal \\ Systems Technology, Inc. \\ Elin Schold-Davis \\ Sister Kenny Institute \\ Erica B. Stern \\ Occupational Therapy \\ University of Minnesota
}

Summary: Jerry I've used the 'track changes' tool so that items I've added are in underlined blue; those I'm suggesting you eliminate are in red with strikethrough

Draft Abstract for Driving Assessment 2001 ConferenceBrain injury due to accident or stroke frequently results in cognitive impairment, reducing an individual's ability to judge driving situations accurately. Rehabilitation professionals typically use a combination of clinical and onroad tests to determine whether an individual is safe to drive. Weighing the safety of the community, the candidate, and the driving evaluator, these on-road tests are often conducted under road, traffic and weather conditions less demanding than those that a driver might face in the "real world," and thus may offer less than complete information regarding the candidate's responses to such real-world driving challenges. Indeed, individuals with mild cognitive deficits may perform adequately under such testing conditions but unsafely when driving challenges increase. Complicating this situation further, those with mild to moderate acquired cognitive impairments may be largely unaware of their own limitations, and thus more intolerant of perceived delays or challenges to their desire to drive again.

Although continuing advances have improved performance and fidelity while significantly reducing costs, most interactive driving simulators remain too expensive for widespread clinical application. In a project funded by the National Center for Medical Rehabilitation Research, National Institutes of Health, we sought to determine, on a pilot basis, whether a low-cost, PCbased driving simulator could provide clinicians with information useful to their efforts to assess the safe ability to drive of individuals with cognitive impairments.

We developed two comprehensive simulator-based driving scenarios, one quite basic and one more challenging, and pilot-tested them on ten subjects - five with moderate cognitive impairments, and five age and sex matched-controls without impairment. The "simple" scenario was developed to match the essential demands of the first half of an existing on-road driving evaluation; the "complex" scenario was based on the second half of the on-road evaluation into 
which more demanding, but still common, driving challenges were integrated. Road types, lane widths, pavement markings, traffic signals, horizontal and vertical curvature, and the proximal built environment were all created in simulation to provide a convincing generic representation of the on-road test. Challenges incorporated into the "complex" phase of the scenario, which were absent from the "simple" phase, included traffic events such as: cross-traffic failing to stop at a STOP sign; pedestrians crossing the driver's path; vehicles suddenly pulling out in front of the subject from the road shoulder; opposing thru traffic appearing suddenly from behind slower moving vehicles as the subject attempted to turn left; slower moving lead vehicles causing passing decisions; traffic streams forcing gap acceptance decisions; etc.

Results from the simulator were compared to results from the on-road evaluation. In addition, data gathered from subject exit interviews was used to judge simulator verisimilitude and efficacy in changing self-awareness of deficit. Because the cognitive impairments associated with brain injury often reduce the individual's awareness of his or her own limitations, we looked at evidence that performance on the simulator could contribute to an individual's own understanding of his or her driving strengths and weaknesses.

The results of the pilot study will lead to an enhancement of simulator capabilities, and to a comprehensive clinical trial at multiple sites. This paper will present the findings of this pilot investigation and an overview of the expanded clinical study. 